\title{
Covid-19: ibuprofen should not be used for managing symptoms, say doctors and scientists
}

\author{
Michael Day
}

London

Scientists and senior doctors have backed claims by France's health minister that people showing symptoms of covid-19 should use paracetamol (acetaminophen) rather than ibuprofen, a drug they said might exacerbate the condition.

The minister, Oliver Veran, tweeted on Saturday 14 March that people with suspected covid-19 should avoid anti-inflammatory drugs. "Taking anti-inflammatory drugs (ibuprofen, cortisone

...) could be an aggravating factor for the infection. If you have a fever, take paracetamol," he said.

His comments seem to have stemmed in part from remarks attributed to an infectious diseases doctor in south west France. She was reported to have cited four cases of young patients with covid-19 and no underlying health problems who went on to develop serious symptoms after using non-steroidal anti-inflammatory drugs (NSAIDs) in the early stage of their symptoms. The hospital posted a comment saying that public discussion of individual cases was inappropriate.

But Jean-Louis Montastruc, a professor of medical and clinical pharmacology at the Central University Hospital in Toulouse, said that such deleterious effects from NSAIDS would not be a surprise given that since 2019, on the advice of the National Agency for the Safety of Medicines and Health Products, French health workers have been told not to treat fever or infections with ibuprofen.

Experts in the UK backed this sentiment. Paul Little, a professor of primary care research at the University of Southampton, said that there was good evidence "that prolonged illness or the complications of respiratory infections may be more common when NSAIDs are used-both respiratory or septic complications and cardiovascular complications."

He added, "The finding in two randomised trials that advice to use ibuprofen results in more severe illness or complications helps confirm that the association seen in observational studies is indeed likely to be causal. Advice to use paracetamol is also less likely to result in complications."

Ian Jones, a professor of virology at the University of Reading, said that ibuprofen's anti-inflammatory properties could "dampen down" the immune system, which could slow the recovery process. He added that it was likely, based on similarities between the new virus (SARS-CoV-2) and SARS I, that covid-19 reduces a key enzyme that part regulates the water and salt concentration in the blood and could contribute to the pneumonia seen in extreme cases. "Ibuprofen aggravates this, while paracetamol does not," he said.

Charlotte Warren-Gash, associate professor of epidemiology at the London School of Hygiene and Tropical Medicine, said: "For covid-19, research is needed into the effects of specific NSAIDs among people with different underlying health conditions. In the meantime, for treating symptoms such as fever and sore throat, it seems sensible to stick to paracetamol as first choice."

Rupert Beale, an infectious diseases researcher at the Francis Crick Institute, had a warning on cortisone, however. "Patients taking cortisone or other steroids should not stop them except on advice from their doctor," he said.

The complex role that the immune system might play in covid-19 disease has been underscored by reports that Swiss drug company Roche has secured approval from China for its anti-inflammation drug Actemra (tocilizumab) to treat patients developing severe complications from covid-19.

Some doctors in Italy, including Paolo Ascierto of the Pascale Hospital in Naples, claim that they have had success treating severely ill patients with the drug, which blocks the key inflammatory molecule interleukin-6.

There is speculation that the drug might prevent fatal "cytokine storms," in which the immune system of seriously ill patients can cause organ failure. 